[Agr. Biol. Chem., Vol. 34, No. 2, p. 274 281, 1970]

\title{
Production of O-Succinyl-L-homoserine by Auxotrophic Mutants of Aerobacter aerogenes
}

\author{
By Hiroshi Kase, Kiyoshi Nakayama and Shukuo Kinoshita \\ Tokyo Research Laboratory, Kyowa Hakko Kogyo Co., Ltd., \\ Machida-shi, Tokyo \\ Received July 14, 1969
}

\begin{abstract}
Ten of Nineteen methionine-requiring mutants isolated from Aerobacter aerogenes ATCC 8308 by treatment with $\mathrm{N}$-methyl- $\mathrm{N}^{\prime}$-nitro- $\mathrm{N}$-nitrosoguanidine were found to accumulate in a culture broth a large amount of O-succinyl-L-homoserine (OSH) which was an intermediate in the biosynthesis of methionine in Escherichia coli and Salmonella typhimurium. OSH was isolated from the culture broth and identified by the behavior in paper chromatography, elementary analysis, melting point, optical density and infrared spectrum. Among these mutants, A. aerogenes KY 7056 which responds to methionine, homocysteine or systathionine was used to investigate culture conditions for $\mathrm{OSH}$ production. The amount of $\mathrm{OSH}$ accumulation reached a level of $8.36 \mathrm{mg} / \mathrm{ml}$ with the medium containing $10 \%$ fructose and $1 \%$ ammonium sulfate. Addition of L-homoserine $(10 \mathrm{mg} / \mathrm{ml})$ increased the amount of OSH accumulation to a level of $15.8 \mathrm{mg} / \mathrm{ml}$. Methionine or cystathionine suppressed the accumulation of OSH. Addition of $\delta$-hydroxylysine to the fermentation medium almost abolished the OSH accumulation.
\end{abstract}

O-Succinyl-L-homoserine (OSH) has recently been found to be an intermediate in the biosynthesis of methionine in Escherichia coli ${ }^{1-31}$ and Salmonella typhimurium ${ }^{3)}$ (Fig. 1). In the course of the studies on the production of amino acids using microbial auxotrophic mu- tants, a large amount of OSH was found to be accumulated by methionine-requiring mutants of Aerobacter aerogenes. The accumulation of $\mathrm{OSH}$ by microbial mutant has been reported only in E. coli and S. typhimurium. Therefore, the isolation and identification of $\mathrm{OSH}$
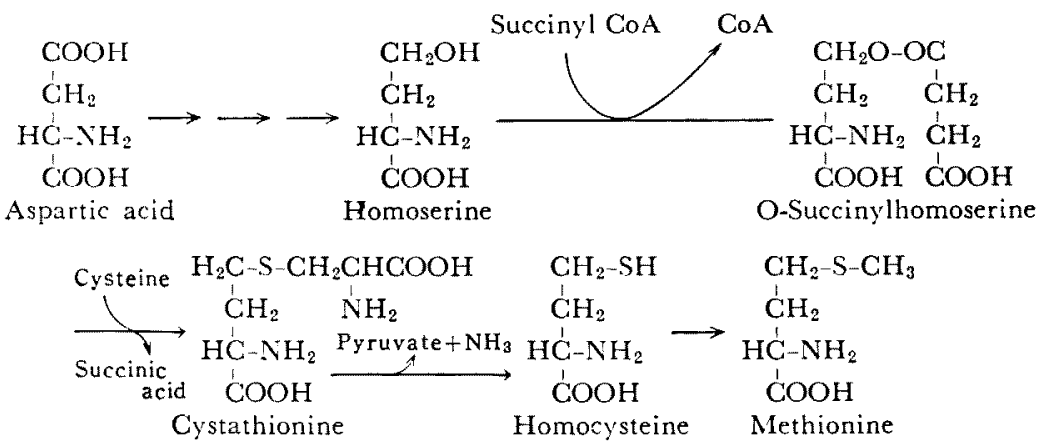

FIG. 1. Pathway of Methionine Synthesis in Escherichia coli and Salmonella typhimurium

1) R. J. Rowbury, J. Gen. Microbiol., 28, 5P (1964) (1962).

2) R. J. Rowbury and D. D. Woods, ibid., 36, 341
3) R. J. Rowbury, J. Gen. Microbiol., 37, 171 
from the culture broth were attempted. Some cultural conditions for the production of $\mathrm{OSH}$ by the mutant were also investigated.

\section{MATERIALS AND METHODS}

Microorganism. Nineteen methionine-requiring mutants were isolated from $A$. aerogenes ATCC 8308 by treatment with $\mathrm{N}$-methyl-N'-nitro- $\mathrm{N}$-nitrosoguanidine, according to the procedure of Adelberg et al.4. Ten of them accumulated $\mathrm{OSH}$ in a culture broth. Among these mutants, $A$. aerogenes KY 7056 was mainly employed for the investigation of fermentation condition for $\mathrm{OSH}$ production.

Culture method. Thirty milliliters of a seed medium in a $250 \mathrm{ml}$ Erlenmyer flask was inoculated with one loopful of organism grown on a bouillon agar slant, and incubated on a rotatry shaker operated at 220 rpm. After incubation for $24 \mathrm{hr}$ at $30^{\circ} \mathrm{C}, 1 \mathrm{ml}$ of this seed culture was transferred into a large test tube $(25 \mathrm{~mm} \times 19 \mathrm{~cm})$ containing $10 \mathrm{ml}$ of a fermentation medium. This test tube was incubated at $30^{\circ} \mathrm{C}$ on a test tube shaker for $72 \mathrm{hr}$. The composition of the medium is described in the footnote of each table and figure. The composition of the seed medium was as follows; $2 \%$ glucose, $1 \%$ peptone, $1 \%$ yeast extract and $0.25 \%$ sodium chloride $(\mathrm{pH} 7.4)$.

Growth experiment. A large test tube containing $10 \mathrm{ml}$ medium was inoculated with one drop of cell suspension from bouillon agar slant (finally, ca. $10^{5}$ cells $/ \mathrm{ml}$ ), and incubated at $30^{\circ} \mathrm{C}$ on a test tube shaker. After $24 \mathrm{hr}$, the optical density of the culture broth at a wave length of $660 \mathrm{~m} \mu$ was read using a Tokyo Koden colorimeter.

Analysis. OSH, and the other amino acids were estimated by colorimetry using ninhydrin after paper chromatographical separation. The paper chromatography was performed on Toyo Roshi No. 50 paper using the following solvent system; n-butanol-acetic acid-water $(4: 1: 2)$. For the estimation of growth in a fermentation medium containing calcium carbonate, $1 \mathrm{ml}$ of $6 \mathrm{~N} \mathrm{HCl}$ was added to $1 \mathrm{ml}$ aliquot of the broth to dissolve the calcium carbonate, and the optical density was measured after dilution $1: 10$.

4) E. A. Adelberg, M. Mandel and G. C. C. Chen, Biochem. Biophys. Res. Commun., 18, 788 (1965).

\section{RESULTS}

\section{Isolation and identification}

When some of the methionine-requiring mutants of $A$.aerogenes were incubated in the medium with limited quantities of methionine, an unknown compound was noticed to be accumulated, which showed a ninhydrin positive spot at $R f 0.35$ when chromatographed on paper in the solvent mixture described in the section, Materials and Methods. The compound gave a yellow brown color spot (which later turned purple) on heating with ninhydrin. The compound was very unstable in mild alkaline solution, but more stable in mild acid solution. After heating the broth adjusted to $4 \mathrm{~N}$ in hydrochloric acid concentration at $100^{\circ} \mathrm{C}$ for $2 \mathrm{hr}$, the spot corresponding to the compound was no more detected on paper chromatogram, while a new spot corresponding to homoserine appeared. These properties agreed closely with those of OSH described in the paper by Rowbury. ${ }^{2,31}$ Then, the attempt was made to isolate OSH from the culture broth. The isolation procedure follow-

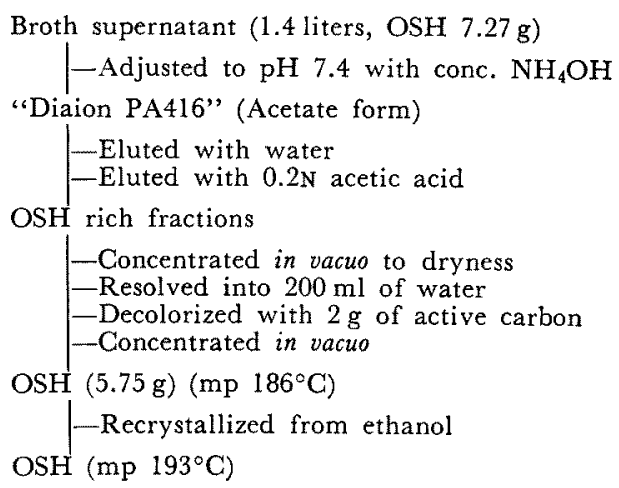

FIG. 2. Isolation of OSH from Broth Supernatant.

ed that by Rowbury ${ }^{3)}$ (Fig. 2). The broth was centrifuged, and the supernatant was adjusted to $\mathrm{pH} 7.4$ with conc. ammonia, and chromatographed on a column of strongly basic anion exchange resin, Diaion PA 416, in the acetate form. Elution was performed 


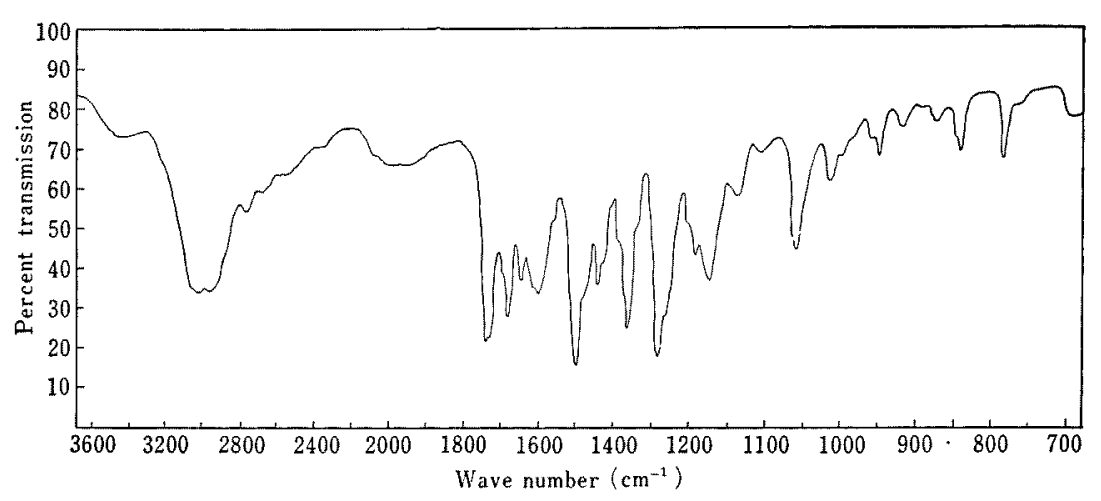

FIG. 3. Infrared Absorption Spectrum of OSH.

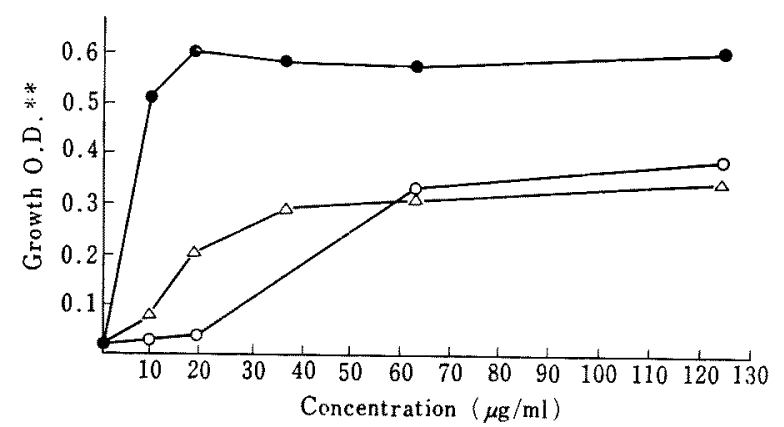

FIG. 4. Growth Response of $A$. aerogenes KY 7056 to Homocysteine, Cystathionine and Methionine.

O-O DL-Homocysteine, $\Delta-\triangle$ DL-Cystathionine

L-Methionine

Medium; glucose $10 \mathrm{~g}, \mathrm{NH}_{4} \mathrm{H}_{2} \mathrm{PO}_{4} 1 \mathrm{~g}, \mathrm{KCl} 0.2 \mathrm{~g}, \mathrm{MgSO}_{4}$.

$7 \mathrm{H}_{2} \mathrm{O} \quad 0.2 \mathrm{~g}$, trace element solution* $1 \mathrm{ml}$, distilled water

1 liter $(\mathrm{pH} 7.2)$.

* $\mathrm{Na}_{2} \mathrm{~B}_{4} \mathrm{O}_{7} \cdot 10 \mathrm{H}_{2} \mathrm{O} 88 \mathrm{mg},\left(\mathrm{NH}_{4}\right)_{6} \mathrm{Mo}_{7} \mathrm{O}_{27} \cdot 4 \mathrm{H}_{2} \mathrm{O} 37 \mathrm{mg}$,

$\mathrm{ZnSO}_{4} \cdot 7 \mathrm{H}_{2} \mathrm{O} 8.8 \mathrm{mg}, \mathrm{CuSO}_{4} \cdot 5 \mathrm{H}_{2} \mathrm{O} 270 \mathrm{mg}, \mathrm{MnCl}_{2} \cdot 4 \mathrm{H}_{2} \mathrm{O}$

$7.2 \mathrm{mg}, \mathrm{FeCl}_{3} \cdot 6 \mathrm{H}_{2} \mathrm{O} 970 \mathrm{mg}$, distilled water 1 liter.

** optical density

with water, then with $0.2 \mathrm{~N}$ acetic acid. $\mathrm{OSH}$ was eluted with $0.2 \mathrm{~N}$ acetic acid and separated from the other amino acids, homoserine and valine, which were accumulated in small amounts in the culture broth. The OSH rich fractions were combined and evaporated to dryness under reduced pressure. The residue was dissolved in water. The solution was treated with active carbon, and concentrated in vacuo, to crystallize OSH. The product was recrystallized from ethanol. The data of the elementary analysis was essentially identical with theoretical value; Found: $\mathrm{H}, 6.13 ; \mathrm{C}$, 44.05; $\mathrm{N}, 6.22$. Calcd. for $\mathrm{C}_{8} \mathrm{H}_{13} \mathrm{O}_{6} \mathrm{~N}: \mathrm{H}, 5.94$; $\mathrm{C}, 43.83$; N, 6.39\%. Melting point $\left(193^{\circ} \mathrm{C}\right)$, optical rotation, $[\alpha]_{\mathrm{D}}^{20}+7.8\left(c=1.0, \mathrm{H}_{2} \mathrm{O}\right)$, and infrared spectrum (Fig. 3) are in good agreement with those in the paper by M. Flavin et al. ${ }^{51}$

5) M. Flavin and C. Slaughter, Biochemistry, 4, 5) M. Flavi
1370 (1965). 
2. Growth response to methionine, homocysteine and cystathionine

As shown in Fig. 4, A. aerogenes KY 7056 responded to methionine, homocysteine or cystathionine. No growth was obtained when methionine was replaced by either cystine or cysteine. The genetic block must, therefore, occur between OSH and cystathionine.

3. Effect of methionine concentration on $\mathrm{OSH}$ accumulation

As shown in Fig. 5, the lower were the methionine levels in fermentation media, the

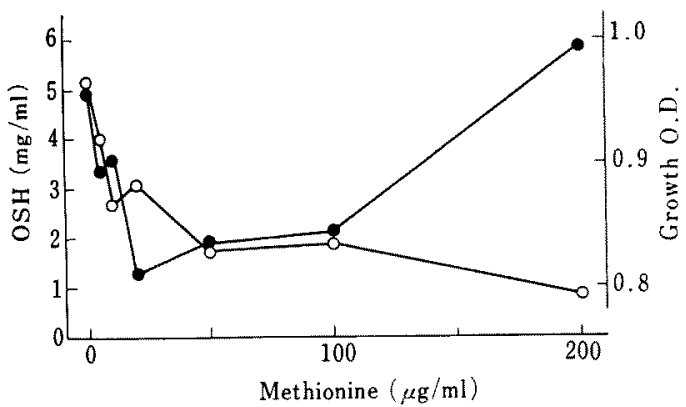

FIG. 5. Effect of Methionine on OSH Accumulation.

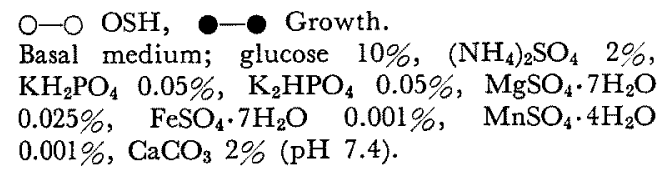

better were $\mathrm{OSH}$ yields. The highest yield was obtained without added methionine. It is assumed that suitable amount of methionine to produce OSH was transferred from a seed culture to a fermentation medium. Excess of methionine suppressed the accumulation of OSH.

4. Effect of carbon source on OSH production

Seven sugars and soluble starch were investigated at 5 and $10 \%$ levels to determine the most favorable carbon source for $\mathrm{OSH}$ production. As shown in Table I, fructose, sorbitol, mannitol, lactose and sucrose gave high yields. Soluble starch seemed to be hardly utilized by this organism. With glucose or xylose, accumulation was moderately good.
TABLE I. EFFECT OF CARBON SOURCE ON OSH ACCUMULATION

\begin{tabular}{|c|c|c|c|c|}
\hline & $\%$ & $\mathrm{pH}$ & O.D. & $\mathrm{OSH} \mathrm{mg} / \mathrm{ml}$ \\
\hline Glucose & $\begin{array}{r}5 \\
10\end{array}$ & $\begin{array}{l}7.6 \\
7.5\end{array}$ & $\begin{array}{l}0.615 \\
0.855\end{array}$ & $\begin{array}{l}3.29 \\
4.73\end{array}$ \\
\hline Fructose & $\begin{array}{r}5 \\
10\end{array}$ & $\begin{array}{l}7.0 \\
6.7\end{array}$ & $\begin{array}{l}0.720 \\
0.970\end{array}$ & $\begin{array}{l}7.00 \\
6.40\end{array}$ \\
\hline Xylose & $\begin{array}{r}5 \\
10\end{array}$ & $\begin{array}{l}7.0 \\
5.8\end{array}$ & $\begin{array}{l}0.700 \\
1.075\end{array}$ & $\begin{array}{l}5.47 \\
4.97\end{array}$ \\
\hline Lactose & $\begin{array}{r}5 \\
10\end{array}$ & $\begin{array}{l}5.6 \\
4.9\end{array}$ & $\begin{array}{l}0.770 \\
0.995\end{array}$ & $\begin{array}{l}4.41 \\
6.02\end{array}$ \\
\hline Mannitol & $\begin{array}{r}5 \\
10\end{array}$ & $\begin{array}{l}7.5 \\
5.7\end{array}$ & $\begin{array}{l}0.748 \\
1.050\end{array}$ & $\begin{array}{l}4.71 \\
6.18\end{array}$ \\
\hline Sorbitol & $\begin{array}{r}5 \\
10\end{array}$ & $\begin{array}{l}6.7 \\
6.9\end{array}$ & $\begin{array}{l}0.795 \\
1.050\end{array}$ & $\begin{array}{l}7.55 \\
5.40\end{array}$ \\
\hline Sucrose & $\begin{array}{r}5 \\
10\end{array}$ & $\begin{array}{l}7.4 \\
7.3\end{array}$ & $\begin{array}{l}0.698 \\
0.965\end{array}$ & $\begin{array}{l}4.78 \\
6.19\end{array}$ \\
\hline Soluble starch & $\begin{array}{r}5 \\
10\end{array}$ & $\begin{array}{l}7.4 \\
5.8\end{array}$ & $\begin{array}{l}0.259 \\
0.400\end{array}$ & $\begin{array}{l}1.69 \\
1.41\end{array}$ \\
\hline
\end{tabular}

5. Effect of inorganic nitrogen source on OSH production

TAble II. EfFect of Nitrogen SOURCE ON OSH ACCUMUlation

\begin{tabular}{lcccc}
\hline & $\%$ & $\mathrm{pH}$ & O.D. & OSH $\mathrm{mg} / \mathrm{ml}$ \\
\hline & 0.5 & 6.1 & 0.835 & 5.86 \\
$\left(\mathrm{NH}_{4}\right)_{2} \mathrm{SO}_{4}$ & 1.0 & 6.7 & 0.915 & 5.03 \\
& 2.0 & 7.6 & 0.855 & 4.55 \\
& 0.4 & 5.8 & 0.815 & 7.21 \\
$\mathrm{NH}_{4} \mathrm{Cl}$ & 0.8 & 6.1 & 0.945 & 6.55 \\
& 1.6 & 6.9 & 0.940 & 5.00 \\
& 0.5 & 5.3 & 0.488 & 4.72 \\
$\left(\mathrm{NH}_{4}\right)_{2} \mathrm{HPO}_{4}$ & 1.0 & 6.6 & 0.668 & 4.23 \\
& 2.0 & 5.8 & 0.355 & 1.68 \\
$\mathrm{NH}_{4} \mathrm{NO}_{3}$ & 0.3 & 7.3 & 0.850 & 4.75 \\
& 0.6 & 7.0 & 0.928 & 3.91 \\
$\mathrm{KNO}_{3}$ & 1.2 & 7.2 & 0.800 & 4.93 \\
& 0.4 & 6.5 & 0.335 & 0.43 \\
$\mathrm{CH}_{3} \mathrm{COONH}_{4}$ & 0.8 & 5.4 & 0.228 & - \\
& 1.5 & 5.4 & 0.223 & - \\
$\mathrm{Urea}$ & 0.6 & 7.1 & 0.795 & 2.41 \\
& 2.3 & 6.3 & 0.705 & 1.22 \\
& 0.2 & 5.6 & 0.458 & 0.08 \\
& 0.5 & 7.8 & 0.570 & 4.72 \\
& 1.0 & 7.5 & 0.800 & 3.52 \\
& & & & 0.82 \\
\hline
\end{tabular}

Basal medium; glucose $10 \%, \mathrm{KH}_{2} \mathrm{PO}_{4} 0.05 \%, \mathrm{~K}_{2} \mathrm{HPO}_{4}$ $0.05 \%, \mathrm{MgSO}_{4} \cdot 7 \mathrm{H}_{2} \mathrm{O} 0.025 \%, \mathrm{FeSO}_{4} \cdot 7 \mathrm{H}_{2} \mathrm{O} 0.001 \%$, $\mathrm{MnSO}_{4} \cdot 4 \mathrm{H}_{2} \mathrm{O} 0.001 \%, \mathrm{CaCO}_{3} 2 \%$ (pH 7.4). 
TABLE III. EFFECT OF CARBon SOURCE AND AMMONIUM SULFATE ON OSH ACCUMULATION

\begin{tabular}{|c|c|c|c|c|c|}
\hline & $\%$ & $\underset{\%}{\left(\mathrm{NH}_{4}\right)_{2} \mathrm{SO}_{4}}$ & $\mathrm{pH}$ & O.D. & $\underset{\mathrm{mg} / \mathrm{ml}}{\mathrm{OSH}}$ \\
\hline \multirow{2}{*}{ Fructose } & 10 & $\begin{array}{l}0.5 \\
1.0 \\
2.0\end{array}$ & $\begin{array}{l}4.6 \\
4.9 \\
5.0\end{array}$ & $\begin{array}{l}0.665 \\
0.880 \\
0.800\end{array}$ & $\begin{array}{l}5.86 \\
8.36 \\
4.14\end{array}$ \\
\hline & 5 & $\begin{array}{l}0.5 \\
1.0 \\
2.0\end{array}$ & $\begin{array}{l}5.6 \\
6.1 \\
6.2\end{array}$ & $\begin{array}{l}0.605 \\
0.740 \\
0.660\end{array}$ & $\begin{array}{l}4.55 \\
5.81 \\
7.53\end{array}$ \\
\hline \multirow{2}{*}{ Sorbitol } & 10 & $\begin{array}{l}0.5 \\
1.0 \\
2.0\end{array}$ & $\begin{array}{l}4.8 \\
5.0 \\
5.6\end{array}$ & $\begin{array}{l}0.750 \\
0.753 \\
0.880\end{array}$ & $\begin{array}{l}4.56 \\
5.44 \\
4.42\end{array}$ \\
\hline & 5 & $\begin{array}{l}0.5 \\
1.0 \\
2.0\end{array}$ & $\begin{array}{l}5.4 \\
6.1 \\
6.4\end{array}$ & $\begin{array}{l}0.643 \\
0.618 \\
0.708\end{array}$ & $\begin{array}{l}5.30 \\
4.82 \\
5.53\end{array}$ \\
\hline
\end{tabular}

Basal medium; $\mathrm{KH}_{2} \mathrm{PO}_{4} \quad 0.05 \%, \mathrm{~K}_{2} \mathrm{HPO}_{4} 0.05 \%$, $\mathrm{MgSO}_{4} \cdot 7 \mathrm{H}_{2} \mathrm{O} 0.025 \%, \mathrm{FeSO}_{4} \cdot 7 \mathrm{H}_{2} \mathrm{O} 0.001 \%, \mathrm{MnSO}_{4} \cdot$ $4 \mathrm{H}_{2} \mathrm{O} 0.001 \%, \mathrm{CaCO}_{3} 2 \%(\mathrm{pH} 7.4)$.

TABLE IV. EFFECT OF INORGanic SALTS ON OSH ACCUMULATION

\begin{tabular}{cllll}
\hline $\mathrm{KH}_{2}^{\mathrm{KHO}_{2} \mathrm{PO}_{4}{ }^{+}}$ & $\begin{array}{c}\mathrm{MgSO}_{2} \\
7 \mathrm{H}_{2} \mathrm{O} \\
\%\end{array}$ & $\mathrm{gH}$ & O.D. & $\begin{array}{c}\mathrm{OSH} \\
\mathrm{mg} / \mathrm{ml}\end{array}$ \\
\hline & 0.0125 & 5.1 & 0.760 & 3.63 \\
0.0063 & 0.025 & 5.2 & 0.723 & 3.30 \\
& 0.05 & 5.2 & 0.758 & 3.26 \\
& 0.1 & 5.1 & 0.750 & 3.45 \\
& 0.2 & 5.1 & 0.745 & 3.21 \\
& 0.0125 & 5.0 & 0.850 & 5.75 \\
0.0125 & 0.025 & 4.9 & 0.880 & 5.36 \\
& 0.05 & 4.9 & 0.850 & 5.63 \\
& 0.1 & 5.0 & 0.860 & 6.29 \\
& 0.2 & 5.2 & 0.885 & 6.77 \\
0.025 & 0.0125 & 6.0 & 0.885 & 6.53 \\
& 0.025 & 5.8 & 0.955 & 7.41 \\
& 0.05 & 5.1 & 1.015 & 7.40 \\
& 0.1 & 5.0 & 0.990 & 7.44 \\
& 0.2 & 5.3 & 1.015 & 7.92 \\
0.05 & 0.0125 & 6.2 & 0.850 & 6.20 \\
& 0.025 & 6.1 & 0.950 & 7.35 \\
& 0.05 & 5.1 & 0.975 & 5.30 \\
& 0.1 & 5.8 & 1.033 & 6.63 \\
& 0.2 & 5.6 & 1.030 & 6.97 \\
\hline
\end{tabular}

a) $\mathrm{KH}_{2} \mathrm{PO}_{4}$ and $\mathrm{K}_{2} \mathrm{HPO}_{4}$ were used in $1: 1$ ratio on weight basis. The concentration in the table represents the level of each phosphate.

Basal medium; fructose $10 \%,\left(\mathrm{NH}_{4}\right)_{2} \mathrm{SO}_{4} 1 \%, \mathrm{FeSO}_{4}$. $7 \mathrm{H}_{2} \mathrm{O} 0.001 \%, \mathrm{MnSO}_{4} \cdot 4 \mathrm{H}_{2} \mathrm{O} 0.001 \%, \mathrm{CaCO}_{3} 2 \%$, (pH 7.4).
Various nitrogen sources were tested for their effects on $\mathrm{OSH}$ production, at the concentrations equal to $0.5,1.0$ and $2.0 \%$ ammonium sulfate on a nitrogen basis. Ammonium salts, and urea gave high yields, and potassium nitrate was found to be unutilized by this organism (Table II). These results were obtained with the medium containing $10 \%$ glucose. Then, experiments were carried out to determine the favorable combination of carbon source and ammonium sulfate and maximum yield $(8.36 \mathrm{mg} / \mathrm{ml})$ was obtained with the medium containing $10 \%$ fructose and $1 \%$ ammonium sulfate (Table III).

\section{Effect of inorganic salts on $\mathrm{OSH}$ production}

The effect of the concentration of phosphate and magnesium ion on $\mathrm{OSH}$ accumulation was studied. The proper ranges of the concentration of dipotassium hydrogen phosphate and potassium dihydrogen phosphate were each $0.0125 \sim 0.05 \%$ and those of magnesium sulfate were $0.025 \sim 0.2 \%$ (Table IV).

\section{Effect of amino acids, vitamines, and precursors on OSH accumulation}

The effect of 24 natural amino acids on $\mathrm{OSH}$ production was investigated. A part of the results is presented in Table V. Addition of homoserine was found to increase $\mathrm{OSH}$ accumulation markedly. Ten milligrams per millilitter of homoserine led to the OSH accumulation of $15.8 \mathrm{mg} / \mathrm{ml}$. Addition of Llysine or $\alpha, \varepsilon$-diaminopimelic acid at low levels enhanced OSH accumulation, while the addition at high levels led to the accumulation of cadaverine, a catabolite of lysine. By addition of L-arginine, glycine, L-glutamic acid, L-glutamine, or L-asparagine, $\mathrm{OSH}$ accumulation was stimulated. High concentration of L-cystine, L-cysteine, L-aspartic acid, L-proline, $\mathrm{L}$-serine, or L-leucine inhibited $\mathrm{OSH}$ production. Addition of $\alpha$-aminobutyric acid at high levels led to the decrease in $\mathrm{OSH}$ production with concurrent accumulation of homoserine and isoleucine.

Besides these natural amino acids, some 
TABlE V. EFFEct OF Amino Acids ON OSH ACcumulation

\begin{tabular}{|c|c|c|c|c|c|c|c|c|c|}
\hline & $\mathrm{mg} / \mathrm{ml}$ & $\mathrm{pH}$ & O.D. & $\begin{array}{l}\mathrm{OSH} \\
\mathrm{mg} / \mathrm{ml}\end{array}$ & & $\mathrm{mg} / \mathrm{ml}$ & $\mathrm{pH}$ & O.D. & $\underset{\mathrm{mg} / \mathrm{ml}}{\mathrm{OSH}}$ \\
\hline \multirow[t]{2}{*}{ L-Homoserine } & $\begin{array}{l}0.2 \\
1 \\
5\end{array}$ & $\begin{array}{l}7.0 \\
7.0 \\
6.4\end{array}$ & $\begin{array}{l}0.645 \\
0.655 \\
0.748\end{array}$ & $\begin{array}{r}6.44 \\
7.53 \\
8.69\end{array}$ & \multirow[t]{2}{*}{ L-Cystine } & $\begin{array}{l}0.2 \\
1 \\
5\end{array}$ & $\begin{array}{l}7.2 \\
6.0 \\
5.8\end{array}$ & $\begin{array}{l}0.710 \\
0.820 \\
0.580\end{array}$ & $\begin{array}{l}5.11 \\
4.14 \\
0.47\end{array}$ \\
\hline & 10 & & & 15.80 & & 0.2 & 7.0 & 0.703 & 5.21 \\
\hline \multirow[t]{2}{*}{ L-Lysine } & $\begin{array}{l}0.2 \\
1\end{array}$ & $\begin{array}{l}5.8 \\
6.4\end{array}$ & $\begin{array}{l}0.730 \\
0.715\end{array}$ & $\begin{array}{l}7.45 \\
7.51\end{array}$ & \multirow[t]{2}{*}{ L-Cysteine } & 1 & $\begin{array}{l}5.5 \\
5.8\end{array}$ & $\begin{array}{l}0.845 \\
0.720\end{array}$ & $\begin{array}{l}4.15 \\
1.11\end{array}$ \\
\hline & 5 & 5.1 & 0.945 & 5.21 & & 0.2 & 7.1 & 0.708 & 5.59 \\
\hline $\mathrm{DAP}^{a)}$ & $\begin{array}{l}0.2 \\
1\end{array}$ & $\begin{array}{l}6.4 \\
5.1\end{array}$ & $\begin{array}{l}0.730 \\
0.890\end{array}$ & $\begin{array}{l}6.73 \\
4.11\end{array}$ & \multirow[t]{3}{*}{ L-Serine } & 1 & $\begin{array}{l}7.1 \\
5.1\end{array}$ & $\begin{array}{l}0.730 \\
0.905\end{array}$ & $\begin{array}{l}5.68 \\
3.82\end{array}$ \\
\hline L-Arginine & 0.2 & 6.4 & 0.780 & 6.65 & & 10 & 5.1 & 0.975 & 1.57 \\
\hline L-Rूg & 1 & 5.7 & 0.895 & 6.47 & & 0.2 & 7.0 & 0.675 & 3.87 \\
\hline Glycine & $\begin{array}{l}0.2 \\
1\end{array}$ & $\begin{array}{l}7.0 \\
5.8\end{array}$ & $\begin{array}{l}0.660 \\
0.885\end{array}$ & $\begin{array}{l}6.76 \\
6.67\end{array}$ & L-Leucine & $\begin{array}{r}1 \\
5 \\
10\end{array}$ & $\begin{array}{l}7.2 \\
7.0 \\
6.2\end{array}$ & $\begin{array}{l}0.740 \\
0.795 \\
0.805\end{array}$ & $\begin{array}{l}3.37 \\
2.19 \\
1.73\end{array}$ \\
\hline L-Glutamic acid & $\begin{array}{l}0.2 \\
1\end{array}$ & $\begin{array}{l}7.2 \\
7.1\end{array}$ & $\begin{array}{l}0.695 \\
0.775\end{array}$ & $\begin{array}{l}6.86 \\
6.60\end{array}$ & \multirow[b]{2}{*}{ L-Aspartic acid } & 0.2 & $\begin{array}{l}0.2 \\
7.0\end{array}$ & 0.705 & 4.58 \\
\hline L-Proline & $\begin{array}{l}0.2 \\
1\end{array}$ & $\begin{array}{l}5.8 \\
5.6\end{array}$ & $\begin{array}{l}0.905 \\
0.950\end{array}$ & $\begin{array}{l}6.64 \\
2.96\end{array}$ & & 1 & $\begin{array}{l}6.7 \\
7.0\end{array}$ & $\begin{array}{l}0.740 \\
0.780\end{array}$ & $\begin{array}{l}4.75 \\
1.63\end{array}$ \\
\hline L-Glutamine & $\begin{array}{l}0.2 \\
1\end{array}$ & $\begin{array}{l}6.3 \\
5.8\end{array}$ & $\begin{array}{l}0.745 \\
0.780\end{array}$ & $\begin{array}{l}6.64 \\
6.66\end{array}$ & \multirow[t]{2}{*}{$\begin{array}{l}\text { DL- } \alpha \text {-Aminobutyric } \\
\quad \text { acid }\end{array}$} & $\begin{array}{l}0.2 \\
1 \\
5\end{array}$ & $\begin{array}{l}7.1 \\
6.2 \\
6.6\end{array}$ & $\begin{array}{l}0.700 \\
0.785 \\
0.815\end{array}$ & $\begin{array}{l}5.52 \\
4.34 \\
2.66\end{array}$ \\
\hline \multirow{2}{*}{ L-Asparagine } & 0.2 & 6.1 & 0.683 & 7.01 & & 10 & 5.8 & 0.313 & - \\
\hline & 1 & 6.0 & 0.770 & 4.66 & Control & - & 6.3 & 0.630 & 4.30 \\
\hline
\end{tabular}

a) $\alpha$, $\varepsilon$-Diaminopimelic acid.

Basal medium; glucose $10 \%,\left(\mathrm{NH}_{4}\right)_{2} \mathrm{SO}_{4} 1 \%, \mathrm{KH}_{2} \mathrm{PO}_{4} 0.05 \%, \mathrm{~K}_{2} \mathrm{HPO}_{4} 0.05 \%, \mathrm{MgSO}_{4} \cdot 7 \mathrm{H}_{2} \mathrm{O} 0.025 \%, \mathrm{FeSO}_{4}$. $7 \mathrm{H}_{2} \mathrm{O} 0.001 \%, \mathrm{MnSO}_{4} \cdot 4 \mathrm{H}_{2} \mathrm{O} 0.001 \%, \mathrm{CaCO}_{3} 2 \%(\mathrm{pH} 7.4$ ).

TABle VI. EFFECT OF SOME AMINo ACIDS ON OSH ACCUMULATION

\begin{tabular}{|c|c|c|c|c|c|}
\hline & $\mathrm{mg} / \mathrm{ml}$ & $\mathrm{pH}$ & O.D. & $\begin{array}{c}\mathrm{OSH} \\
\mathrm{mg} / \mathrm{ml}\end{array}$ & $\begin{array}{l}\text { Other amino } \\
\text { acids } \mathrm{mg} / \mathrm{ml}\end{array}$ \\
\hline Cystathionine & $\begin{array}{l}0.2 \\
1 \\
5 \\
10\end{array}$ & $\begin{array}{l}6.0 \\
6.9 \\
6.0 \\
6.0\end{array}$ & $\begin{array}{l}0.915 \\
1.010 \\
1.065 \\
0.950\end{array}$ & $\begin{array}{l}1.28 \\
\operatorname{tr} . \\
\operatorname{tr} . \\
\text { tr. }\end{array}$ & $\begin{array}{l}\text { val } \pm \sim+ \\
\text { val }++ \\
\text { Met } 0.63 \\
\text { Met } 1.78\end{array}$ \\
\hline $\begin{array}{c}\delta \text {-Hydroxy- } \\
\text { lysine }\end{array}$ & $\begin{array}{l}0.2 \\
1 \\
5 \\
10\end{array}$ & $\begin{array}{l}5.4 \\
6.9 \\
6.8 \\
5.8\end{array}$ & $\begin{array}{l}0.910 \\
0.925 \\
0.850 \\
0.710\end{array}$ & $\begin{array}{l}5.38 \\
4.85 \\
0.51 \\
0.30\end{array}$ & \\
\hline Thialysine & $\begin{array}{l}0.2 \\
1 \\
5 \\
10\end{array}$ & $\begin{array}{l}5.8 \\
5.2 \\
5.2 \\
5.9\end{array}$ & $\begin{array}{l}0.875 \\
0.860 \\
0.840 \\
0.715\end{array}$ & $\begin{array}{l}5.16 \\
5.21 \\
4.61 \\
4.76\end{array}$ & \\
\hline Lanthionine & $\begin{array}{c}0.2 \\
1 \\
5 \\
10\end{array}$ & $\begin{array}{l}6.5 \\
6.5 \\
6.3 \\
6.3\end{array}$ & $\begin{array}{l}0.940 \\
0.950 \\
1.010 \\
1.060\end{array}$ & $\begin{array}{l}6.14 \\
5.61 \\
6.09 \\
7.76\end{array}$ & \\
\hline Control & - & 6.2 & 0.930 & 5.41 & \\
\hline
\end{tabular}

Basal medium; fructose $10 \%,\left(\mathrm{NH}_{4}\right)_{2} \mathrm{SO}_{4} 1 \%, \mathrm{KH}_{2} \mathrm{PO}_{4}$ $0.05 \%, \mathrm{~K}_{2} \mathrm{HPO}_{4} 0.05 \%, \mathrm{MgSO}_{4} \cdot 7 \mathrm{H}_{2} \mathrm{O} 0.025 \%, \mathrm{FeSO}_{4}$. $7 \mathrm{H}_{2} \mathrm{O} 0.001 \%, \mathrm{MnSO}_{4} \cdot 4 \mathrm{H}_{2} \mathrm{O} 0.001 \%, \mathrm{CaCO}_{3} 2 \%(\mathrm{pH}$ 7.4).
TABLE VII. EFFECT OF SOME AMINo ACIDS ON OSH ACCUMULATION

\begin{tabular}{|c|c|c|c|c|}
\hline & $\mathrm{mg} / \mathrm{ml}$ & $\mathrm{pH}$ & O.D. & $\underset{\mathrm{mg} / \mathrm{ml}}{\mathrm{OSH}}$ \\
\hline D-Lysine & $\begin{array}{l}0.2 \\
1 \\
5 \\
10\end{array}$ & $\begin{array}{l}5.8 \\
5.8 \\
6.4 \\
5.6\end{array}$ & $\begin{array}{l}1.045 \\
0.955 \\
0.910 \\
0.855\end{array}$ & $\begin{array}{l}6.46 \\
7.12 \\
4.05 \\
3.31\end{array}$ \\
\hline $\begin{array}{l}\alpha \text {-Ketobutyric } \\
\text { acid }\end{array}$ & $\begin{array}{l}0.2 \\
1 \\
5 \\
10\end{array}$ & $\begin{array}{l}5.8 \\
6.0 \\
5.9 \\
5.8\end{array}$ & $\begin{array}{l}0.975 \\
0.940 \\
0.361 \\
0.236\end{array}$ & $\begin{array}{l}3.44 \\
5.51 \\
\mp \\
7\end{array}$ \\
\hline nor-Leucine & $\begin{array}{c}0.2 \\
1 \\
5 \\
10\end{array}$ & $\begin{array}{l}5.4 \\
5.8 \\
5.8 \\
5.8\end{array}$ & $\begin{array}{l}0.990 \\
0.398 \\
0.293 \\
0.270\end{array}$ & $\begin{array}{l}\bar{F} \\
\bar{F} \\
F\end{array}$ \\
\hline nor-Valine & $\begin{array}{l}0.2 \\
1 \\
5 \\
10\end{array}$ & $\begin{array}{l}5.3 \\
5.8 \\
5.9 \\
5.8\end{array}$ & $\begin{array}{l}0.920 \\
0.615 \\
0.658 \\
0.292\end{array}$ & $\begin{array}{l}4.93 \\
0.94 \\
0.30 \\
\text { tr. }\end{array}$ \\
\hline Control & - & 6.0 & 0.927 & 3.81 \\
\hline
\end{tabular}

Basal medium; fructose $10 \%,\left(\mathrm{NH}_{4}\right)_{2} \mathrm{SO}_{4} 1 \%, \mathrm{KH}_{2} \mathrm{PO}_{4}$ $0.05 \%, \mathrm{~K}_{2} \mathrm{HPO}_{4} 0.05 \%, \mathrm{MgSO}_{4} \cdot 7 \mathrm{H}_{2} \mathrm{O} 0.025 \%, \mathrm{FeSO}_{4}$. $7 \mathrm{H}_{2} \mathrm{O} 0.001 \%, \mathrm{MnSO}_{4} \cdot 4 \mathrm{H}_{2} \mathrm{O} 0.001 \%, \mathrm{CaCO}_{3} 2 \%(\mathrm{pH}$ 7.4). 
other amino acids including lysine analogues, $\delta$-hydroxylysine, thialysine ( $\mathrm{S}-\beta$-aminoethylcysteine), and diaminopimelic acid analogue, lanthionine, were investigated on their effects on $\mathrm{OSH}$ production. The results are presented in Tables VI and VII. $\delta$-Hydroxylysine suppressed OSH accumulation at high levels, while the influence of thialysine was slight. Although the effect of D-lysine was similar to that of L-lysine, cadaverine accumulation was not noted even at high D-lysine levels. Furthermore, the addition of high levels (1 $10 \mathrm{mg} / \mathrm{ml}$ ) of D-lysine led to the accumulation of small amount of threonine. Lanthionine was found to promote $\mathrm{OSH}$ accumulation to some extent. A methionine precursor, cystathionine, suppressed the $\mathrm{OSH}$ production markedly. Besides, low concentration of cystathionine stimulated the production of valine, and high concentration of it caused the accumulation of methionine. $\alpha$-Ketobutyric acid, nor-valine, and nor-leucine retarded both growth and $\mathrm{OSH}$ production.

Nine vitamines, including thiamine, riboflavine, $p$-aminobenzoic acid, pyridoxine, nicotinic acid, biotin, folic acid, cyanocobalamine and inositol $(0.2 \mu \mathrm{g} / \mathrm{liter}$ and $2 \mu \mathrm{g} / \mathrm{liter})$, fumaric acid and succinic acid $(5 \mathrm{mg} / \mathrm{ml}$ and $10 \mathrm{mg} / \mathrm{ml}$ ) showed slight effect at best.

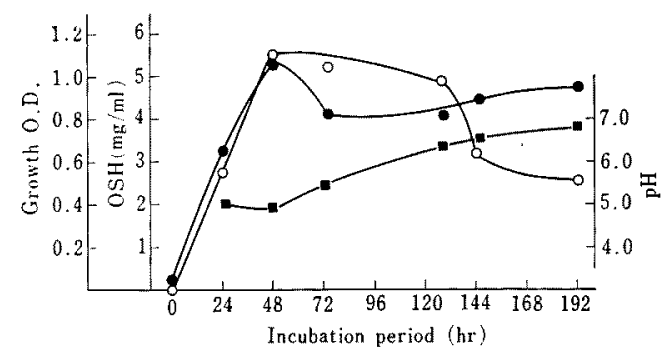

FIG. 6. Time Course of OSH Fermentation.

O-O OSH,

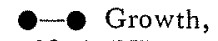

- $\mathbf{p H}$.

Medium; fructose $10 \%,\left(\mathrm{NH}_{4}\right)_{2} \mathrm{SO}_{4} 1 \%, \mathrm{KH}_{2} \mathrm{PO}_{4}$ $0.05 \%, \mathrm{~K}_{2} \mathrm{HPO}_{4} 0.05 \%, \mathrm{MgSO}_{4} \cdot 7 \mathrm{H}_{2} \mathrm{O} 0.025 \%$, $\mathrm{FeSO}_{4} \cdot 7 \mathrm{H}_{2} \mathrm{O} \quad 0.001 \%, \quad \mathrm{MnSO}_{4} \cdot 4 \mathrm{H}_{2} \mathrm{O} 0.001 \%$, $\mathrm{CaCO}_{3} 2 \%$ (pH 7.4).

\section{Changes occurring during fermentation}

An example of the chemical changes occurring during the fermentation under optimal conditions is shown in Fig. 6. Production of OSH paralleled growth, reached a maximum in about $48 \mathrm{hr}$, after which OSH concentration steadily declined with a concurrent rise of $\mathrm{pH}$-value, and rapidly declined after $120 \mathrm{hr}$.

\section{DISCUSSION}

OSH has been found to be accumulated by the mutants of $A$. aerogenes which are blocked between homoserine and cystathionine. This finding indicates that $\mathrm{OSH}$ is an intermediate in methionine biosynthesis in $A$. aerogenes, as has been reported in $E$. coli and $S$. typhimurium. L-Methionine inhibited the formation of OSH (Fig. 5). This fact suggests that the regulation mechanism in methionine synthesis reported in $E$. coli $i^{1 \sim 3 \prime}$ and $S$. typhimurium ${ }^{31}$ functions also in $A$. aerogenes. Addition of cystathionine suppressed the OSH formation, when methionine accumulated. It may be considered that cystathionine inhibited the formation of $\mathrm{OSH}$ after conversion into methionine.

O-Succinylhomoserine transsuccinylase may be speculated to be the step which receives feedback type inhibition by methionine although the evidence supporting this speculation awaits to be obtained. Addition of homoserine increased $\mathrm{OSH}$ accumulation markedly (Table V). This fact indicates that homoserine is a precursor of OSH. In contrast, succinic acid did not stimulate the OSH formation. This organism may be able to supply the amount of succinic acid required endogenously while the formation of homoserine is limited.

Addition of $\alpha$-aminobutyric acid at high levels $(5 \sim 10 \mathrm{mg} / \mathrm{ml})$ led to the decrease in OSH production with the concurrent accumulation of homoserine and isoleucine. The production of isoleucine from $\alpha$-aminobutyric acid has been well documented. ${ }^{67}$ However, it is not clear whether homoserine accumula- 
tion in addition of $\alpha$-aminobutyric acid is due to the decomposition of OSH or to the inhibition by $\alpha$-aminobutyric acid of the reaction between homoserine and OSH.

$\delta$-Hydroxylysine suppressed OSH accumulation at high levels (Table VI). The inhibition of aspartokinase by $\delta$-hydroxylysine in

6) M. Hayashibe, M. Ito, J. Watanabe and T. Uemura, Hakko To Taisha, 2, 100 (1960).

7) I. Chibata, M. Kisumi and Y. Ashikaga, J. Biochem. Microbial. Technol. Eng., 2, 361 (1960).
Streptococcus faecalis has been reported. ${ }^{81}$ Whether similar type of inhibition led to the suppression of OSH accumulation requires further investigation.

During the fermentation, OSH concentration was rapidly declined after $120 \mathrm{hr}$. Although the factor involved is not clear, the possibility that the induction of an anabolic pathway other than the synthetic pathway of methionine may be considered.

8) D. P. Gilboe, J. D. Friede and L. M. Hendersen, J. Bacteriol., 95, 856 (1968). 